\title{
Effect of Double Bond on Fluxional Nature of Cyclopentane: An Evolutionary Leap from Hirsutanes to Capnellanes
}

\author{
Nader Sakhaee $^{1,2 *}$, Sahar Sakhaee ${ }^{3}$, Akbar Mobaraki $^{4}$ and Ahmad Takallou ${ }^{5}$ \\ ${ }^{1}$ Department of mathematics and Natural Sciences, Harris-Stowe State University, St. Louis, MO, 63108, USA \\ ${ }^{2}$ Department of Chemistry, Southern Illinois University Edwardsville, Edwardsville, IL 62025, USA \\ ${ }^{3}$ Islamic Azad University, Mashhad Branch, Mashhad, Iran
}

${ }^{4}$ Department of Chemistry, Tarbiat Modarres University, P.O. Box. 14115-175, Tehran, Iran

${ }^{5}$ Department of Chemistry, Kharazmi University, Tehran, Iran

*Corresponding author: Nader Sakhaee, Department of mathematics and Natural Sciences, Harris-Stowe State University, St. Louis, MO, 63108, USA and Department of Chemistry, Southern Illinois University Edwardsville, Edwardsville, IL 62025, USA

\begin{tabular}{l} 
ARTICLE INFO \\
\hline Received: 嵊 January 18, 2020 \\
Published: 幽 February 03, 2020 \\
\hline
\end{tabular}

Citation: Nader Sakhaee, Sahar Sakhaee, Akbar Mobaraki, Ahmad Takallou. Effect of Double Bond on Fluxional Nature of Cyclopentane: An Evolutionary Leap from Hirsutanes to Capnellanes. Biomed J Sci \& Tech Res 25(2)-2020. BJSTR. MS.ID.0041670.

\begin{abstract}
In an effort to understand the druggable chemical space of sesquiterpenes, conformational flexibility has been a hot research branch. Here the recently proposed spherical conformational landscape was tested to evaluate the effect of introduction of double bonds on conformational mobility in cyclopentane fluxional rings. The SCL model for cyclopentene and cyclopentadiene rings, suggest quiet a remarkable restriction brought up by double bonds in these otherwise fluxional molecules. The focus here will then be on the effect of double bonds on fused sesquiterpenoid structure. Comparisons of two classes of natural products namely hirsutanes and capnellanes, respectively originated from sponges and soft corals with a 100-million-year time window, reveals a clear pattern of mobility for the latter. Authors believe that nature has evolved clever ways to get rid of double bonds in hirsutanes and design more tunable dynamic scaffolds for more potent capnellanes thru ages of evolution. Further ongoing research on the dynamics of these cyclic compounds may reveal fresh guidelines in druggable chemical space.
\end{abstract}

Keywords: Spherical Conformational Landscape Model; Fused Sesquiterpenoid Structure; Fluxional Nature; Hirsutanes; Capnellanes; Ring Coordinates; Druggable Chemical Space

\section{Introduction}

Medicinal plants have long been known to humans. Special plant species had even attracted animals for their potential drug properties. Thanks to our modern chemical technology we can now extract and study the very structure of these biomedical compounds [1-7]. The field has grown into a full-blown rapidly expanding area of chemistry known as natural products. A new and recent off-shoot was the treasure trove of marine natural products with even more potency compared to their terrestrial analogues. Common to both sources, is the need for motion less life forms in

need of physical protection. The ocean has been the evolutionary bench test of life for nearly 4000 million years [8], while pioneer land dwelling species emerged as late as 430 million years. Such a meaningful evolutionary lag could well translate into potency observed for marine natural products compared to their terrestrial analogues. After focusing on a very simple chemical functionality on cyclopentane backbone using SCL model, we shall visit the evolutionary design from hirsutanes to capnellanes over almost a 100 million years from sponges in Neoproterozoic seas to soft corals in early Cambrian seas. 


\section{Methods}

Full conformational analysis in cyclopentene and cyclopentadiene were done using density functional method $\omega \mathrm{b} 97 x d$, in particular, yield reliable results. In some cases, correlational methods like $\mathrm{MP}_{2}$ gave similar or close results to those obtained via DFT/ $\omega b 97 x d$. The only drawback of most local

Table 1: Relative energies of all conformers along ring coordinates with letter code labeling as shown in Figure 1 . All 20 conformers for both cyclopentene and cyclopentadiene are given.

\begin{tabular}{|c|c|c|c|c|c|c|}
\hline Compounds & \multicolumn{6}{|c|}{ Conformers / Energies (Kcal/mol) } \\
\hline \multirow{2}{*}{ Cyclopentane } & $a, a^{\prime}, b, b^{\prime}, c, c^{\prime}, d, d^{\prime}, e, e^{\prime}$ & $u, u^{\prime} . w, w^{\prime}, x, x^{\prime}, y, y^{\prime}, z, z^{\prime}$ & & & & \\
\hline & 0 & 0.5 & & & & \\
\hline \multicolumn{7}{|c|}{ Cyclopentene } \\
\hline & $c, c^{\prime}$ & $d, b, b^{\prime}, d^{\prime}$ & $\mathrm{x}, \mathrm{z}^{\prime}, \mathrm{x}^{\prime}, \mathrm{z}$ & $\mathrm{w}, \mathrm{u}^{\prime} \mathrm{w}^{\prime}, \mathrm{u}$ & $\mathrm{a}, \mathrm{a}^{\prime} \mathrm{e}, \mathrm{e}^{\prime}$ & $\mathrm{y}, \mathrm{y}^{\prime}$ \\
\hline & 0 & 7 & 17 & 2 & 14 & 30 \\
\hline \multicolumn{7}{|c|}{ Cyclopentadiene } \\
\hline & $\mathrm{c}, \mathrm{c}^{\prime}$ & $d, b, b^{\prime}, d^{\prime}$ & $\mathrm{x}, \mathrm{z}^{\prime}, \mathrm{x}^{\prime}, \mathrm{z}$ & $\mathrm{w}, \mathrm{u}^{\prime} \mathrm{w}^{\prime}, \mathrm{u}$ & $a, a^{\prime}$ & $\mathrm{y}, \mathrm{y}^{\prime}$ \\
\hline & & & & & $\mathrm{e}, \mathrm{e}^{\prime}$ & \\
\hline & 7 & 18 & 27 & 9 & 30 & 35 \\
\hline
\end{tabular}

\section{Results and Discussion}

Many reports and pioneering studies have so far focused on conformational analysis of cyclic compounds and in particular cyclopentane molecule alone [14-24]. Conformational landscape model was presented back in 2016, its since been expanded for both cyclohexane and cyclopentane recently. The most prominent feature of the SCL model is its twist and bent ring coordinates which are delicately interconnected. The continuity of this connection can be translated as flexibility and dynamic nature of the compounds often directly reflected in $D / G$ band region of their Raman spectra. Before testing the effect of a double bond functionality its noteworthy to look at the convention used here in brief. Spherical conformational density functional methods, slightly over estimate barriers due to their inability to account for van der Waals attractions in some strained conformers $[9,10]$. The $\omega b 97 x d$ basis set can reliably result in accurate van der Waals modifications. The geometries computations were done at $\omega b 97 x d / 6-311+G(d)$ level $[11,12]$, like 6-311+G(d,p) , which proved almost no better accuracy. using Gaussian g09 package [13]. We also checked larger basis sets

landscape model for parent cyclopentane is shown (Figure 1 left) as a pointed star representation with generic drawing conventions used for the two major classes of dynamic conformers in this fluxional molecule with high gaseous entropy content (letter coded conformers here are used later in Table 1 where relative energies are given). A simpler more informative SCL form containing only ring coordinates and their connections is also shown (Figure 1 middle top). These twist and bent conformers are shown from different viewpoints. (Figure 1 right top). Drawing conventions are all topical views with filled circles and crosses showing upward and downward maximum puckering points respectively. Lighter lines show flanking areas as the plane of the molecule folds up or down.

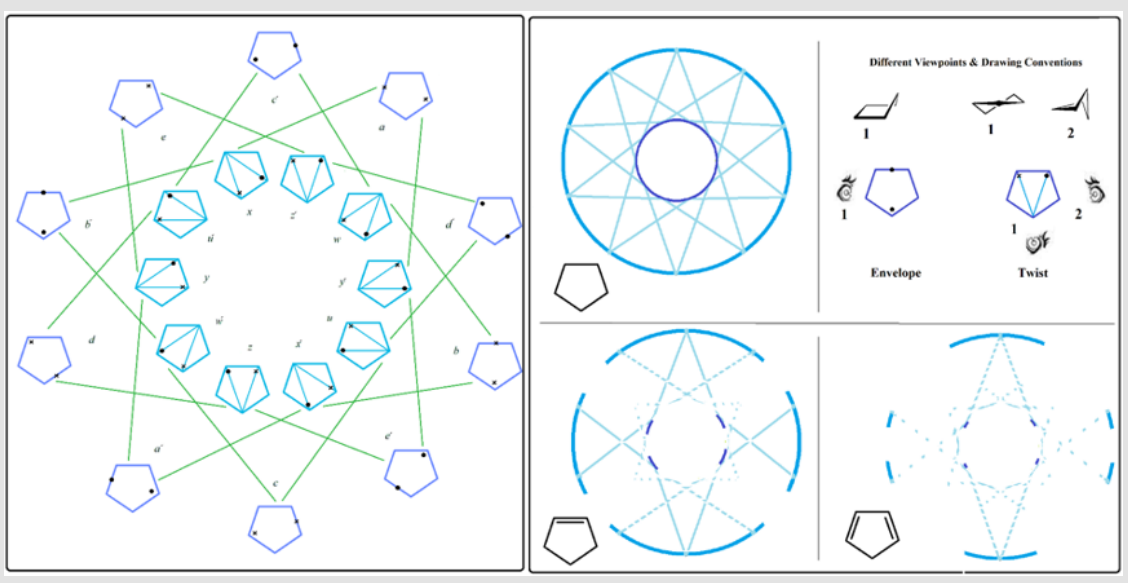

Figure 1: Spherical conformational landscape for cyclopentane star pointed model with conformers shown (left**) the ring coordinate and inter connection without conformers (Top middle). Topical views for twist and bent conformers (Top right). SCL model for cyclopentene and cyclopentadiene (bottom middle and right).

Note: Reprinted from our publication [31] with modifications and extensions to include cyclohexane, copyright 2016 Elsevier publishing Co. 
While Cyclopentane is in constant buzz, introduction of a double bond can restrict this fluxional nature and thus affect its mobile conformational flip flop. With cyclopentene one can see that introduction of a double bond results in a disruption pattern that is most sever in the inner twist ring coordinate which is interestingly the more important ring coordinate to fluxionality of cyclopentane (Figure 1 left bottom). Thus, the double bond functionality can be a serious push back against this fluxional nature and introducing a second double bond proves this to be the case with twist ring being severely restricted and bent ring coordinate also hugely affected. The second double bond almost lead to a conformationally frozen cyclopentane system (Figure 1 left bottom). Such an effect is also evident in D/G band of Raman spectra explained in detail in our previous reports. Table one provides all the energies along the two ring coordinates for both cyclopentene and cyclopentadiene (letters used here are the same that appear in Figure 1).

Natural products have been an inspiration to both synthetic and theoretical organic chemists over the past few decades. Authors believe the impact of ring fusion on the five membered rings has well served evolution a toolbox from which to develop a cast of natural products like hirsutanes, isohirsutanes, capnellanes and silphiperfolanes. Whereas, in a previous study the mobility of cis fusion of cyclopentane rings where discussed, how nature has gained conformational control on these cis fused five membered cyclic compounds is an open question yet to be addressed. This paper briefly explains why two class of these compounds happen to have evolved from an earlier from with double bonds to a more recent and more potent form with no double bonds. Hirsutanes $[25,26]$ had been synthesized by marine sponges as early as 640 MYA, and a generic hirsutane at least has 1 double bond with one $\mathrm{sp}^{2}$ carbon at the fusion point of the two rings. Later with the emergence of earliest soft corals as late as 535 MYA, capnellanes [27-29] were synthesized that are more flexible and when finetuned can be much more potent as well. Capnellanes structures lack the double bond feature and showcase a dynamic curved motion that can be a significant clue as to how flexibility could be finetuned by nature to serve a better more potent bioactivity [30] that's needed for soft coral survival and give them an evolutionary edge. However, a full SCL model for complex three ring systems has not yet been proposed but the telltale signs of how such conformational mobility might appear is presented here with two triple-twist conformers that are ready and cooperatively interconvertible and can well be the most dynamic path in the final SCL model for Capnellanes (Figure 2).
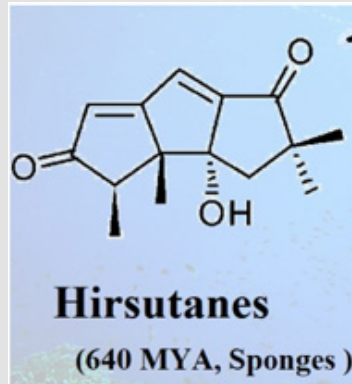

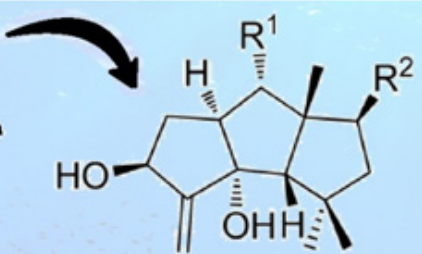

Capnellanes (535 MAY, Corals)
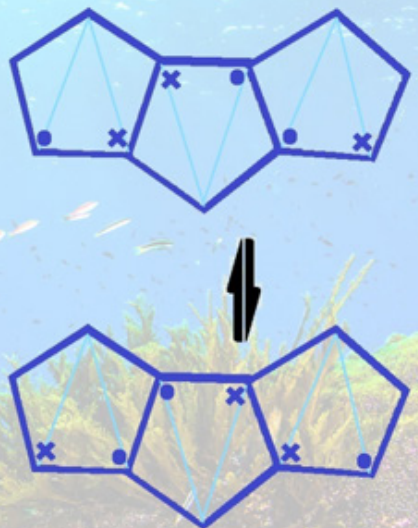

Figure 2: Earlier version of defense chemical produced by sponges back in Neoproterozoic era (left) later more potent sesquiterpenoid natural product synthesized by soft corals in Cambrian seas as late as 535 MYA (middle). Dynamic flip flop in later more potent version marine natural product, capnellanes, that benefits the lack of a double bond in its structure.

\section{Conclusion}

The so called spherical conformational landscape model was used here to test the effect of introduction of double bond functionality on cyclopentane constant flip flop. The disruption was found to be profound and also targets the twist ring coordinate, which is believed to be the crucial ring coordinate to resist any fluxional block or disruption. The drawing convention used in SCL were then used to root out a major dynamic interconversion route in capnellanes. Such dynamic path evidently reveals capnellanes' flexibility compared to hirustanes which are restricted by a presence of a double bond at the fusion point. Not interestingly capnellanes has been the result of evolutionary force over the course of 100 million years and have also reported to be much more potent than hirustanes. Patterns like this could not only deepen our understanding of marine natural products and their evolutionary course but also help us mimic natures ability to introduce much more potent drug compounds in near future.

\section{References}

1. Spivey AC, Weston M, Woodhead S (2002) Celastraceae sesquiterpenoids: biological activity and synthesis. Chemical Society Reviews 31: 43-59. 
2. Yang XL, Zhang JZ, Luo DQ (2012) The taxonomy, biology and chemistry of the fungal Pestalotiopsis genus. Natural product reports 29:622-641.

3. Kreuger MRO, Grootjans S, Biavatti MW, Vandenabeele P, D'Herde K (2012) Sesquiterpene lactones as drugs with multiple targets in cancer treatment: focus on parthenolide. Anti-cancer drugs 23(9): 883-896.

4. Tanasova M, Sturla SJ (2012) Chemistry and biology of acylfulvenes: sesquiterpene-derived antitumor agents. Chemical Reviews 112: 35783610.

5. Durán Peña MJ, Ares JMB, Hanson JR, Collado IG, Hernández Galán R (2015) Biological activity of natural sesquiterpenoids containing a gemdimethylcyclopropane unit. Natural product reports 32: 1236-1248.

6. Elissawy AM, El Shazly M, Ebada SS, Singab AB, Proksch P (2015) Bioactive terpenes from marine-derived fungi. Marine drugs 13(4): 1966-1992.

7. Bartikova H, Hanusova V, Skalova L, Ambroz M, Bousova I (2014) Antioxidant, pro-oxidant and other biological activities of sesquiterpenes. Current topics in medicinal chemistry 14(22): 2478-2494.

8. Thornburg CC, Zabriskie TM, Mc Phail KL (2010) Deep-sea hydrothermal vents: potential hot spots for natural products discovery? Journal of natural products 73(3): 489-499.

9. Halgren TA (1996) Merck molecular force field. II. MMFF94 van der Waals and electrostatic parameters for intermolecular interactions. Journal of Computational Chemistry 17(5-6): 520-552.

10. Giesen DJ, Gu MZ, Cramer CJ, Truhlar DG (1996) A universal organic solvation model. The Journal of organic chemistry 61: 8720-8721.

11. Shao Y, Molnar LF, Jung Y, Kussmann J, Ochsenfeld C, et al. (2006) Advances in methods and algorithms in a modern quantum chemistry program package. Physical Chemistry Chemical Physics 8: 3172-3191.

12. Frisch M, Trucks G, Schlegel HB, Scuseria GE, Robb MA, et al. (2009) Gaussian 09, revision a. 02, gaussian, Inc, Wallingford, CT, England, pp. 200.

13. Hratchian HP, Parandekar PV, Raghavachari K, Frisch MJ, Vreven T (2008) QM: QM electronic embedding using Mulliken atomic charges: Energies and analytic gradients in an ONIOM framework. The Journal of chemical physics 128(3): 034107.

14. Hoffmann RW (2000) Conformation design of open-chain compounds. Angewandte Chemie International Edition 39(12): 2054-2070.

15. Ashwell G, Morell AG (1974) The role of surface carbohydrates in the hepatic recognition and transport of circulating glycoproteins. Advances in enzymology and related areas of molecular biology 41: 99-128.

16. Kilpatrick JE, Pitzer KS, Spitzer R (1974) The thermodynamics and molecular structure of cyclopentane1. Journal of the American Chemical Society 69(10): 2483-2488.

17. Pitzer KS, Donath WE (1993) Conformations and strain energy of cyclopentane and its derivatives, Molecular Structure and Statistical Thermodynamics: Selected Papers of Kenneth S Pitzer. World Scientific. pp. 98-103.

\section{ISSN: 2574-1241}

DOI: $10.26717 /$ BJSTR.2020.25.004170

Nader Sakhaee. Biomed J Sci \& Tech Res

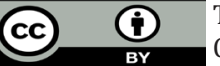

This work is licensed under Creative

Commons Attribution 4.0 License

Submission Link: https://biomedres.us/submit-manuscript.php
18. Wertz D (1969) Far-Infrared Absorption Spectrum and Pseudorotation of the Thiacyclopentane Molecule. The Journal of Chemical Physics 51: 2133-2136.

19. Allinger NL, Hirsch JA, Miller MA, Tyminski IJ, Van Catledge FA (1968) Conformational analysis. LX. Improved calculations of the structures and energies of hydrocarbons by the Westheimer method. Journal of the American Chemical Society 90(5): 1199-1210.

20. Green W, Harvey A, Greenhouse J (1971) Spectroscopic Determination of the Pseudorotation Barrier in Selenacyclopentane. The Journal of Chemical Physics 54: 850-856.

21. Marino J, Long JK (1988) New strategies for annulations: a highly convergent and stereoselective synthesis of an octahydronaphthalene synthon for dihydrocompactin. Journal of the American Chemical Society 110: 7916-7917.

22. Laane J (1969) Far-Infrared Spectrum and the Barrier to Pseudorotation of Silacyclopentane. The Journal of Chemical Physics 50: 1946-1951.

23. Brügger G, Frey HM, Steinegger P, Balmer F, Leutwyler S (2011) Accurate determination of the structure of cyclohexane by femtosecond rotational coherence spectroscopy and ab initio calculations. The Journal of Physical Chemistry A 115: 9567-9578.

24. Kowalewski P, Frey HM, Infanger D, Leutwyler S (2015) Probing the structure, pseudorotation, and radial vibrations of cyclopentane by femtosecond rotational Raman coherence spectroscopy. The Journal of Physical Chemistry A 119: 11215-11225.

25. TAKAZAwA H, KASHINO S (1991) Incarnal. A new antibacterial sesquiterpene from basidiomycetes. Chemical and pharmaceutical bulletin 39: 555-557.

26. Li HJ, Chen T, Xie YL, Chen WD, Zhu XF, et al. (2013) Isolation and structural elucidation of chondrosterins $\mathrm{F}-\mathrm{H}$ from the marine fungus Chondrostereum sp. Marine drugs 11(2): 551-558.

27. Ayanoglu E, Gebreyesus T, Beechan C, Djerassi C, Kaisin M (1978) Terpenoids LXXV. $\Delta 9$ (12)-capnellene, a new sesquiterpene hydrocarbon from the soft coral capnellaimbricata. Tetrahedron Letters 19: 16711674.

28. Kaisin M, Sheikh Y, Durham L, Djerassi C, Tursch B, et al. (1974) Capnellane-a new tricyclic sesquiterpene skeleton from the soft coral capnella imbricate. Tetrahedron Letters 15: 2239-2242.

29. Karlsson $\mathrm{R}$ (1977) The structure and absolute configuration of $\Delta 9$ (12)-capnellene-3 $\beta, 8 \beta, 10 \alpha$-triol. Acta Crystallographica Section B: Structural Crystallography and Crystal Chemistry 33: 1143-1147.

30. Hoang BX, Sawall Y, Al-Sofyani A, Wahl M (2015) Chemical versus structural defense against fish predation in two dominant soft coral species (Xeniidae) in the Red Sea. Aquatic Biology 23: 129-137.

31. Sakhaee N, Jalili S, Darvish F (2016) Spherical conformational landscape shed new lights on fluxional nature of cyclopentane and its derivatives, confirmed by their Raman spectra. Computational and Theoretical Chemistry 1090: 193-202.

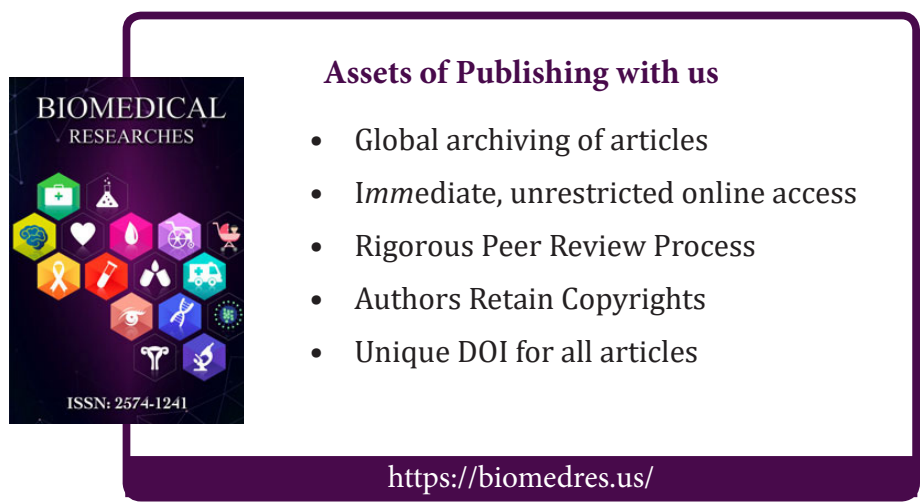

